\title{
Cerebral Responses to Stationary Emotional Stimuli Measured by fMRI in Women with Persistent Postural-Perceptual Dizziness
}

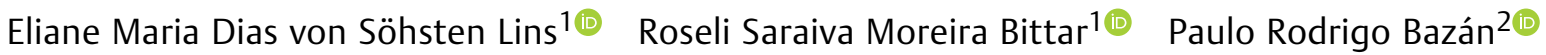

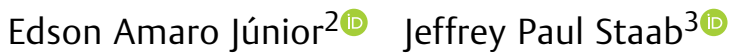

${ }^{1}$ Department of Otorhinolaryngology, Hospital das Clínicas, Universidade de São Paulo, São Paulo, SP, Brazil

${ }^{2}$ Department of Radiology, Hospital das Clínicas, Universidade de São Paulo, São Paulo, SP, Brazil

${ }^{3}$ Department of Psychiatry, Mayo Clinic, Rochester, USA

\begin{abstract}
Address for correspondence Eliane Maria Dias von Söhsten Lins, MD, PhD, 965/12, Jardim Paulista, São Paulo, SP, 01424001, Brazil (e-mail: elianesohsten@gmail.com).
\end{abstract}

\begin{abstract}
Keywords

- anxiety

- angular gyrus

- anterior cingulate cortex

- chronic dizziness

- functional magnetic resonance imaging

- persistent postural perceptual dizziness
\end{abstract}

Introduction Persistent postural-perceptual dizziness (PPPD) is a functional vestibular disorder characterized by chronic dizziness, unsteadiness, and hypersensitivity to motion. Preexisting anxiety disorders and neurotic personality traits confer vulnerability to PPPD. High anxiety during acute vertigo or dizziness incites it. A functional magnetic resonance imaging ( $\mathrm{FMRI}$ ) study of chronic subjective dizziness found unexpectedly hypoactive responses to vestibular stimulation in cortical regions that integrate threat assessment and spatial perception.

Objective This fMRI study used non-moving, but emotionally charged visual stimuli to investigate the brain's activity of PPPD patients and control subjects.

Methods The participants included 16 women with PPPD and 16 age-matched women who recovered completely from acute episodes of vertigo or dizziness capable of triggering PPPD. Brain responses to positive, neutral, and negative figures from the International Affective Picture System were measured with fMRI and compared between the groups. Dizziness handicap, anxiety, and depression were assessed with validated questionnaires.

Results Between group analyses: Participants with PPPD showed reduced activity in anterior cingulate cortex and increased activity in left angular gyrus in response to negative versus positive stimuli, which was not observed in recovered individuals. Within group analyses: Participants with PPPD had increased activity in visuospatial areas (parahippocampal gyrus, intraparietal sulcus) in negative versus positive and negative versus neutral contrasts, whereas recovered individuals had increased activity in anxiety regions (amygdala, orbitofrontal cortex).

Conclusion Patients with PPPD may be more attuned to spatial elements than to the content of emotionally charged visual stimuli. received

April 3, 2020

accepted

July 23, 2020

published online

September 24, 2020
DOI https://doi.org/ 10.1055/s-0040-1716572. ISSN 1809-9777. (c) 2020. Fundação Otorrinolaringologia. All rights reserved.

This is an open access article published by Thieme under the terms of the Creative Commons Attribution-NonDerivative-NonCommercial-License, permitting copying and reproduction so long as the original work is given appropriate credit. Contents may not be used for commercial purposes, or adapted, remixed, transformed or built upon. (https://creativecommons.org/ licenses/by-nc-nd/4.0/)

Thieme Revinter Publicações Ltda., Rua do Matoso 170, Rio de Janeiro, RJ, CEP 20270-135, Brazil 


\section{Introduction}

Persistent postural-perceptual dizziness (PPPD) is a chronic functional vestibular disorder that manifests with waxing and waning dizziness, unsteadiness, and swaying or rocking (nonspinning) vertigo lasting 3 months or longer. ${ }^{1,2}$ Upright posture, active or passive movement, and exposure to motion-rich environments exacerbate these core physical symptoms of PPPD. Medical and psychiatric conditions that cause vertigo, unsteadiness, or dizziness, such as peripheral vestibular disorders, vestibular migraine, panic attacks or generalized anxiety with prominent dizziness, autonomic dysregulation, mild traumatic brain injury, and cardiac dysrhythmias may trigger PPPD in vulnerable individuals, particularly those with anxiety diatheses. $^{2,3}$ In tertiary neurotologic settings, PPPD is the second most common cause of vestibular symptoms and a frequent cause of long-term disability. ${ }^{2,3}$ Up to three-quarters of patients with PPPD may develop comorbid anxiety or depressive disorders over the course of their illness, but PPPD also may present as a sole diagnosis. ${ }^{3}$ The Committee for Classification of Vestibular Disorders of the Bárány Society, the premier international scientific society for neurotologic research, developed formal diagnostic criteria for $\mathrm{PPPD},{ }^{2}$ based on clinical observations dating back 145 years and systematic studies from the last 3 decades. The World Health Organization included PPPD in its draft of the $11^{\text {th }}$ edition of the International Classification of Diseases. ${ }^{1}$

The contemporary predecessors of PPPD were phobic postural vertigo (PPV), ${ }^{4}$ space-motion discomfort (SMD), ${ }^{5}$ visual vertigo (VV), ${ }^{6}$ and chronic subjective dizziness (CSD). ${ }^{7}$ Initial descriptions of each of these entities included symptoms of chronic or recurrent dizziness and hypersensitivity to motion stimuli, but emphasized different provocative factors and relationships to psychological morbidity. Present-day discussions about these syndromes reprised the century-old debates about the relative roles of vestibular, visual, and anxietyrelated processes in spatial perception and locomotion. ${ }^{3,8}$

Persistent postural-perceptual dizziness is a functional vestibular disorder, ${ }^{2,3}$ not a structural or psychiatric illness. A detailed hypothesis of possible pathophysiologic mechanisms ${ }^{2,3,9}$ was derived from numerous investigations of PPV, SMD, VV, and CSD. Functional changes in postural control were surmised from studies of patients with PPV who were found to have stance and gait mechanics that closely resembled those of normal individuals under conditions of postural threat (e.g., stiffened stance and prolonged two-footed support while walking). ${ }^{9}$ A functional shift in multi-sensory information processing was postulated from investigations of patients with chronic VV who were found to favor visual inputs over vestibular and somatosensory stimuli for determining spatial orientation, a phenomenon known as visual dependence. ${ }^{10}$ Roles for anxiety-related factors were hypothesized from findings that patients with $\mathrm{CSD}^{11,12}$ and PPPD $^{13}$ were significantly more likely than control subjects to possess an anxiety diathesis of neurotic personality traits ${ }^{11-13}$ or a personal or family history of anxiety disorders, ${ }^{14}$ whereas individuals with more resilient traits were protected from developing PPV. ${ }^{15}$ Furthermore, a highly anxious, body-vigilant response to triggering events was found to be a key initial step in generating chronic dizziness. ${ }^{10,16,17}$ Taken together, these data indicate that PPPD is a condition in which a tendency toward heightened anxiety and an initially fearful response to acute vestibular or balance symptoms lead to persistent functional changes in locomotor mechanics and space-motion information processing. Importantly, PPPD is not the somatic manifestation of a psychiatric disorder, as it may exist without any overt psychopathology. 2,3,8 Nonetheless, the evidence that anxiety-related processes may confer risk and serve as inciting factors for PPPD suggests a need for greater understanding of the state of anxiety systems in the brain and their effects on space-motion information processing in patients with PPPD.

Four previous neuroimaging investigations bear on this topic. ${ }^{18-21}$ In the first study, Indovina et $\mathrm{al}^{18}$ used fMRI to record brain responses to sound-evoked vestibular stimulation in normal individuals. Neuroticism, measured by the Neuroticism-Extraversion-Openness Personality InventoryRevised (NEO-PI-R), ${ }^{22}$ correlated positively with activity in the cerebellar fastigium, pons, and the $\mathrm{V} 2$ region of the visual cortex, and negatively with activity in the supramarginal gyrus, a vestibular cortical region. Introversion correlated positively with activity in the amygdala. Neuroticism also correlated positively with connectivity between the cerebellar fastigium and the amygdala, pons and amygdala, V2 and inferior frontal gyrus, and supramarginal and inferior frontal gyri, whereas introversion correlated negatively with connectivity between the amygdala and inferior frontal gyrus. In the second study, Riccelli et al ${ }^{19}$ used fMRI to measure brain responses to visual stimuli from a virtual reality rollercoaster in normal individuals, comparing activity and connectivity during vertical (more threatening) versus horizontal (less threatening) portions of the ride. In the vertical versus horizontal contrast, neuroticism correlated positively with activity in the posterior insula and retroinsula and with connectivity between left posterior insula and left inferior frontal gyrus, left retroinsula and right temporal-parietal junction, and left retroinsula and right and left amygdalae. Thus, anxiety-related personality traits were associated with increased activity in cortical and subcortical vestibular, visual, and anxiety regions of the brain and greater connectivity among these regions in normal individuals in response to both vestibular and visual motion stimuli, ${ }^{18,19}$ and also with a shift to greater activity in visual than vestibular cortical areas. ${ }^{18}$ These findings fit with clinical data on the potential roles of anxiety-related factors in predisposing individuals to PPPD and inciting its development. ${ }^{2,3}$

Given these findings, the first neuroimaging investigations of patients with $\mathrm{CSD}^{20}$ and $\mathrm{PPV}^{21}$ yielded unexpected results. Indovina et $\mathrm{al}^{20}$ the team that investigated the response of normal individuals to sound-evoked vestibular stimulation, used the same fMRI protocol to study patients with CSD. Curiously, they found that patients with CSD had lesser, not greater, activations of vestibular cortical areas, anterior insula, inferior frontal gyrus, anterior cingulate cortex, and hippocampus as well as lesser, not greater, connectivity between vestibular cortical areas, frontal cortical regions, and the hippocampus than healthy control 
subjects matched for neuroticism and introversion. In a voxel-based morphometry study of patients with PPV, Wurthmann et $\mathrm{al}^{21}$ reported reduced gray matter volumes in many of these same regions. These results indicate that cortical networks responsible for high-level integration of threat assessment and space-motion perception might be less responsive and less well connected in patients with CSD and PPV than in normal individuals. These four neuroimaging investigations present an intriguing mix of findings. In normal individuals, a moderately loud, but otherwise nonthreatening, vestibular stimulus increased activity and connectivity from the cerebellum and brainstem to the amygdala, and from portions of the primary visual cortex to the inferior frontal gyrus. A potentially threatening visual stimulus increased activity and connectivity more rostrally, from the amygdala to the insula and to portions of the vestibular cortex and inferior frontal gyrus. In patients with CSD, by contrast, sound-evoked vestibular stimulation decreased activity and connectivity across a broad range of rostral regions from the hippocampus, insula and anterior cingulate cortex to portions of the vestibular cortex and inferior frontal gyrus. Thus, more information about the state of anxiety systems in the brains of patients with PPPD and their effects on processing of space-motion information is needed to assimilate these results into a cogent model of the disorder.

We designed the present fMRI study to complement this previous work. Recognizing the anxiety diathesis associated with PPPD, we were interested in whether patients with this disorder might have an increase in reactivity to threatening stimuli in general, not just to inputs containing predominantly space-motion information. To test this hypothesis, we measured brain responses to a standardized set of pleasant, neutral, and threatening pictures. ${ }^{23}$ We presented the pictures in a stationary manner to measure participants' responses to their content without the confounding influence of movement, which could have been particularly provocative for patients with PPPD. We were most interested in the activity of brain regions involved in threat assessment, but we also examined activity in areas responsible for processing vestibular and visual inputs and space-motion perception. We chose a comparison group of age- and sexmatched patients who had recovered from acute vestibular events of the type that are capable of triggering PPPD (i.e., an exposed, but recovered control group) to control for the noxious experience of illnesses that cause acute vertigo or dizziness. We also measured dizziness handicap, state and trait anxiety, and depression in all participants using standardized scales. $^{24-27}$

\section{Methods}

The institutional ethics committee of Clinical Hospital of São Paulo University approved this study, clinical trial number U1111-1144-8754. All participants provided written informed consent before undertaking any investigational procedures.

This was a cross-sectional observational study conducted between May 2012 and December 2014. Female, right-hand- ed subjects, 18 to 60 years old, were recruited from Neurotology Division of ENT Department of Clinical Hospital of São Paulo University if they were diagnosed with PPPD or had recovered fully from a disorder that manifested with acute vestibular symptoms or disturbance of balance. Patients with other neurologic conditions including migraine, as well as other otologic, psychiatric, cardiac, or unstable systemic diseases, motor limitations, uncorrected visual impairments, implanted metallic objects, history of claustrophobia, and those taking psychotropic drugs for any reason were excluded. Sex, handedness, and age restrictions were employed to limit fMRI confounds related to these variables. Persistent postural-perceptual dizziness affects more women than men, with an average age of onset of 40 to 45 years $^{13,28}$; hence the choice of sex and age range restrictions.

Prior to entry into the study, all patients had thorough clinical neurotologic assessments and laboratory testing, including blood tests for common causes of dizziness, pure tone and speech recognition audiometry, impedance testing, electronystagmography, sinusoidal rotary chair testing, and computerized dynamic posturography with the EquiTest Sensory Organization Test (NeuroCom International, Clackamas, OR, USA). Then, they were treated for their neurotologic illnesses as clinically indicated using medications (betahistine, dimenhydrinate, and meclizine as vestibular suppressants; cyclobenzaprine as a cervical muscle relaxant; and statins for metabolic syndromes), canalith repositioning maneuvers for benign paroxysmal positional vertigo, nutritional $^{29,30}$ and hydration countermeasures for dysautonomia and postural hypotension, cervical physiotherapy for neck symptoms, and vestibular habituation to reduce sensitivity to motion stimuli. ${ }^{31}$ Patients who had persistent dizziness despite initial treatment and met the Bárány Society's definition for PPPD, ${ }^{2}$ which was available in draft form at the start of this investigation, constituted the pool of potential subjects with PPPD. Patients who recovered fully from their acute illnesses and were matched for age to the PPPD participants constituted the potential pool of recovered subjects. Of 26 patients in the initial PPPD group, 1 was left-handed, 3 had medical exclusions, and 5 had abnormalities on MRI that could have confounded analyses of fMRI results (e.g., white matter anomalies). Imaging data could not be collected from one enrolled participant because of equipment problems. Of 18 volunteers in the recovered group, 2 were excluded because of anatomical MRI anomalies. That left 16 women in the PPPD group and 16 women in the recovered group for whom data were available.

Before fMRI acquisition, all participants completed validated Portuguese-language versions of the Self-Reporting Questionnaire (SRQ-20), a 20-item screening tool for psychiatric illness, ${ }^{32}$ as well as specific self-reported measures for anxiety and depression, including the 14-item Hospital Anxiety and Depression Scale (HADS-A and HADS-D for anxiety and depression subscales, respectively), ${ }^{24}$ the 20 -item state and trait versions of the State-Trait Anxiety Inventory (STAI-S and STAI$\mathrm{T}$, for the state and trait versions, respectively), ${ }^{25}$ and the $21-$ item Beck Depression Inventory - II (BDI-II). ${ }^{26}$ We measured the severity of dizziness and dizziness-related impairment 
with the Dizziness Handicap Inventory (DHI), ${ }^{27}$ a widely used 25-item self-report of physical and emotional symptoms and functional limitations due to dizziness.

We acquired fMRI images using a Philips Achieva 3.0 Tesla MRI system (Philips Medical Systems, Eindhoven, Netherlands) with a Quasar Dual gradient up to $80 \mathrm{mT} / \mathrm{m}$ and an 8-channel skull coil. We obtained whole brain images using echo-planar imaging with T2-weighted gradient-echo sequences. The acquisition parameters were: time of repetition $=3,000$ milliseconds, echo time $=30$ milliseconds, run time $=450$ second +12 second of dummy scans, thickness $=3.0 \mathrm{~mm}$, gap between slices $=0.3 \mathrm{~mm}$, field of view $240 \times 240 \mathrm{~mm}^{2}$ and matrix $80 \times 80 \mathrm{~mm}^{2}$, with 41 slices in the anterior commissure - posterior commissure plane, resulting in $3 \mathrm{~mm}$ isotropic voxels. The total number of volumes in each run was 150 . All runs were preceded by four "dummy scans" to account for T1 signal decay.

We selected 75 images from the International Affective Picture System (IAPS ${ }^{23}$ and separated them into three groups according to their valence scores: 25 positive, 25 negative, and 25 neutral images. The selected images had similar dominance and arousal values based on standard IAPS scores for women. We presented the images on a 29.5inch projection screen located inside the scanner room using a block fMRI design. Each fMRI experiment consisted of one run of 450 seconds containing 15 epochs ( 5 blocks of each valence condition lasting 30 seconds ( - Fig. 1, top). In each block, 10 IAPS images of the same valence were presented for 2.5 seconds. Each IAPS image was followed by a nonsense image presented for 0.5 second. The nonsense image was constructed from a collage of small square fragments clipped from the 75 selected images (-Fig. 1, bottom). We interspersed the nonsense image to maintain participant engagement and overall level of stimulus complexity.

\section{Data Analysis}

We analyzed fMRI data using the functional MRI of the brain (FMRIB) Software Library version 5.0.1, tool FEAT $6.01 .{ }^{33} \mathrm{We}$ preprocessed images by applying a slice-time and motion correction, spatial smoothing $(5 \mathrm{~mm}$ full width at half maximum), and normalization in the Montreal Neurological Institute standard space. In the first-level analysis for each individual, we used general linear models (GLMs) to detect the variation in blood oxygen level dependent (BOLD) signal associated with blocks of positive and negative images. We included separate independent variables for responses to positive and negative blocks and their time derivatives with the neutral block modeled as baseline. In the second-level analysis, we used mixed effects models to account for variability between subjects. This allowed generalization of the results for the populations studied. Dependent variables were parameters estimated from the first-level GLMs for positive block, negative block, and differences between them. The independent variable was group (PPPD versus Recovered).

We used the Student $t$-test to compare the average BOLD signal within each group for each block type and for block differences. Then, we used the Student $t$-test for independent

\section{A. Positive Image + $\quad$ Neutral Image $\mathbf{0}$ Negative Image}

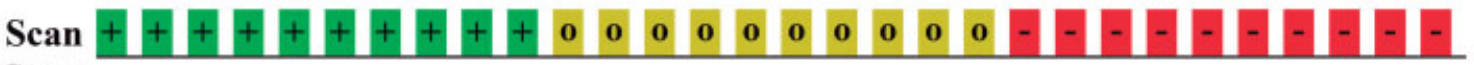 Start}

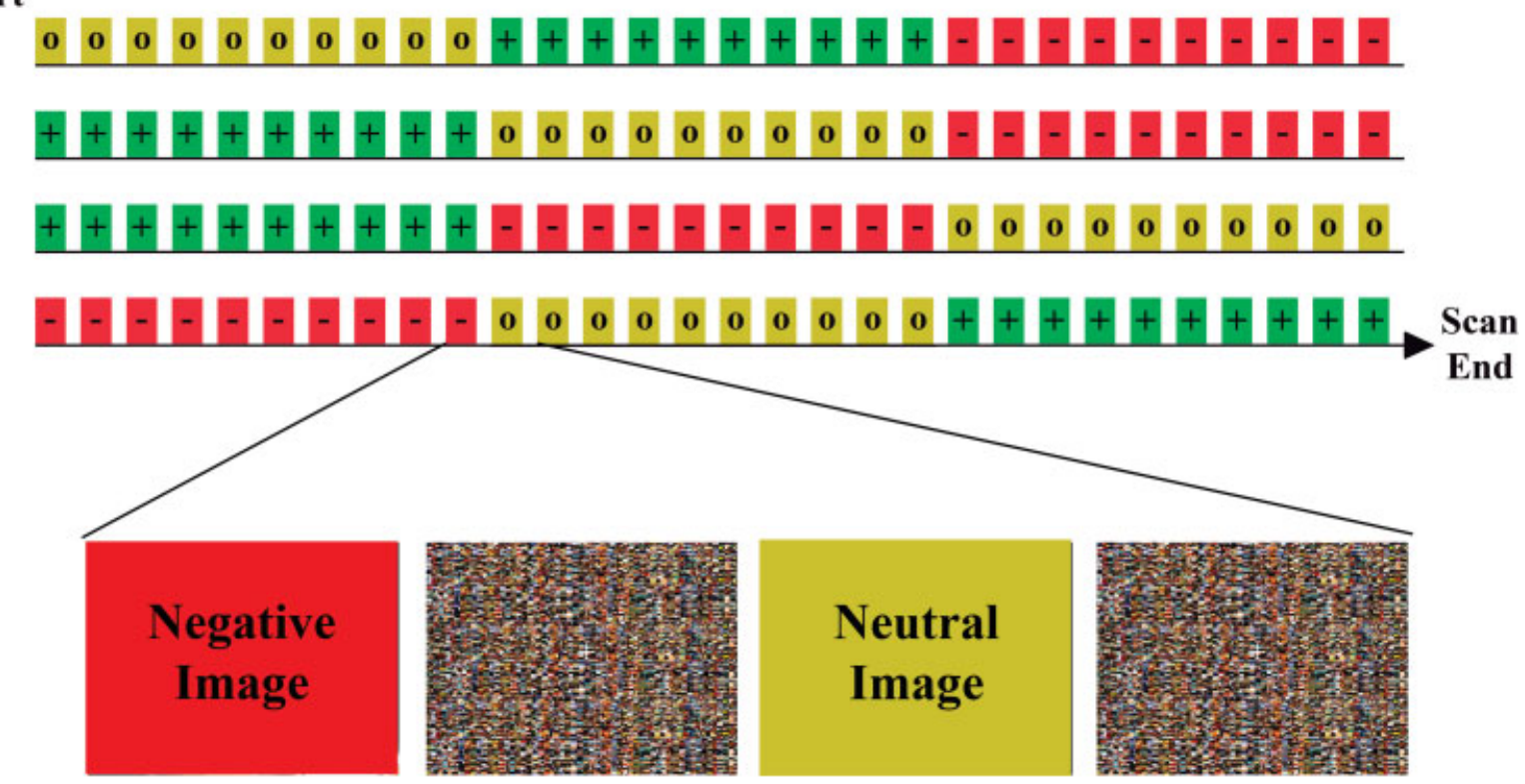

\section{$2.5 \mathrm{~s}$

Fig. 1 Representative scheme of the paradigm: yellow = figures with neutral valence; white = figure of mixed images; red = figures with negative valence; green $=$ figures with positive valence. Block $=$ set of 20 images (10 with positive, negative or neutral valence; 10 equal, from mixed images). 
Table 1 Demographics, dizziness handicap and psychological status of participants

\begin{tabular}{|l|l|l|l|}
\hline & $\begin{array}{l}\text { PPPD } \\
\text { group }\end{array}$ & $\begin{array}{l}\text { Recovered } \\
\text { group }\end{array}$ & $\begin{array}{l}\text { Significance } \\
\text { level }\end{array}$ \\
\hline Age (years) & $44.7 \pm 8.3$ & $46.5 \pm 8.5$ & $p=0.54$ \\
\hline $\begin{array}{l}\text { Dizziness handicap } \\
\text { inventory } \\
\text { (DHI) }\end{array}$ & 52.87 & 10.60 & $p=0.00018^{*}$ \\
\hline $\begin{array}{l}\text { Self-reporting } \\
\text { questionnaire } \\
\text { (SRQ-20) }\end{array}$ & 11.12 & 3.44 & $p<0.00001$ \\
\hline $\begin{array}{l}\text { State-trait anxiety } \\
\text { inventory } \\
\text { trait anxiety (STAI-T) }\end{array}$ & 53.37 & 39.00 & $p<0.000533$ \\
\hline $\begin{array}{l}\text { State-trait anxiety } \\
\text { inventory } \\
\text { state anxiety (STAI-S) }\end{array}$ & 39.00 & 35.07 & $p=0.34$ \\
\hline $\begin{array}{l}\text { Hospital anxiety and } \\
\text { depression scale } \\
\text { anxiety subscale } \\
\text { (HADS-A) }\end{array}$ & 12.87 & 4.06 & $p<0.00001$ \\
\hline $\begin{array}{l}\text { Hospital anxiety and } \\
\text { depression scale } \\
\text { depression subscale } \\
\text { (HADS-D) }\end{array}$ & 7.31 & 2.56 & $p<0.000016$ \\
\hline $\begin{array}{l}\text { Beck depression } \\
\text { inventory } \\
\text { (BDI) }\end{array}$ & 13.67 & 5.00 & $p<0.00001$ \\
\hline
\end{tabular}

Abbreviation: PPPD, persistent postural-perceptual dizziness.

*Mann-Whitney U-test (non-parametric distribution), Student $t$-test for all others

samples to compare differences between groups. To control for false positive results owing to multiple comparisons in the average of each group, we used a threshold Zscore $=3.09, p<0.001$, for each voxel, ${ }^{34}$ and adopted the correction by clusters at a significance level of $p<0.05$. For comparison between groups, we used the less restrictive voxel $\mathrm{Z}$-score $=2.3$ to avoid false negatives results, ${ }^{35}$ while maintaining cluster significance level at $p<0.05$.

Our primary hypothesis was that the PPPD group would show greater reactivity to blocks of negatively-valenced versus positively-valenced stimuli than the Recovered group. We conducted a whole brain analysis and then focused attention on threat assessment regions (e.g., amygdala, limbic nuclei, and peri-limbic cortical areas) followed by regions involved in vestibular and visual information processing and space-motion perception.

\section{Results}

- Table 1 shows comparisons of the demographics and psychiatric status of participants in the PPPD and Recovered groups. The average age of participants in the two groups was similar. Average DHI scores indicated that participants with PPPD were moderately handicapped, whereas individuals in the Recovered group were minimally symptomatic. A significantly greater proportion of participants in the PPPD than in the Recovered group screened positive for psychiatric distress on the SRQ-20. Self-ratings of depression (HADS-D and
Table 2 Causes of initial vestibular or balance symptoms

\begin{tabular}{|l|l|l|}
\hline Initial Disease & $\begin{array}{l}\text { PPPD } \\
\text { group }\end{array}$ & $\begin{array}{l}\text { Recovered } \\
\text { group }\end{array}$ \\
\hline Primary psychiatric disorder & 5 & 0 \\
\hline Metabolic dysfunction & 4 & 7 \\
\hline $\begin{array}{l}\text { Benign paroxysmal } \\
\text { positional vertigo }\end{array}$ & 3 & 4 \\
\hline Costen syndrome & 0 & 1 \\
\hline Xanthine abuse & 0 & 2 \\
\hline $\begin{array}{l}\text { Hormonal } \\
\text { (hypothyroidism) }\end{array}$ & 0 & 1 \\
\hline Menierè disease & 1 & 1 \\
\hline Dysautonomia & 2 & 0 \\
\hline $\begin{array}{l}\text { Proprioceptive } \\
\text { cervical syndrome }\end{array}$ & 1 & 0 \\
\hline Total & 16 & 16 \\
\hline
\end{tabular}

Abbreviation: PPPD, persistent postural-perceptual dizziness.

BDI-II) showed that participants in the PPPD group were on average mildly depressed, whereas those in the Recovered group were not depressed. The PPPD group had a higher average level of trait anxiety (STAI-T) than the Recovered group, but state anxiety scores were mixed with the PPPD group having a significantly higher average score on the HADS-A, but not the STAI-S. - Table 2 lists the illnesses that caused initial vestibular and balance symptoms. More participants in the PPPD group had a psychiatric trigger, whereas more individuals in the Recovered group had a metabolic trigger. Nonetheless, the acute illnesses of all participants in both groups were recognized precipitants of PPPD. 2,3,28,29 Taken together, the demographics, physical illness characteristics, and psychological symptoms of participants in the PPPD group were typical of patients with this disorder and differed in expected ways from those in the Recovered group. ${ }^{2,3,28,29}$

To assess the fidelity of our fMRI results, we reviewed activation patterns in the visual cortex. Participants in both groups showed activation of occipital lobes in response to positive blocks of IAPS figures (-Fig. 2) and activation of the occipital lobes plus superior frontal gyri, right middle and inferior frontal gyri, middle and inferior temporal gyri, borders of the intraparietal sulcus, and cerebellar hemispheres (not midline structures associated with balance) in response to negative blocks (-Fig. $\mathbf{3}$ ). There were no differences between groups in these responses. This indicated that participants in both groups were attentive to the task and registered expected differences between positive and negative blocks. ${ }^{36,37}$

Comparisons between groups identified deactivation of the anterior cingulate cortex in the PPPD group, especially in response to negative stimulus blocks, whereas the Recovered group had little stimulus-induced activity in this region, such that the negative versus positive contrast was significantly different between groups (-Table 3, - Fig. 4, right). In contrast, the PPPD group showed significantly greater 


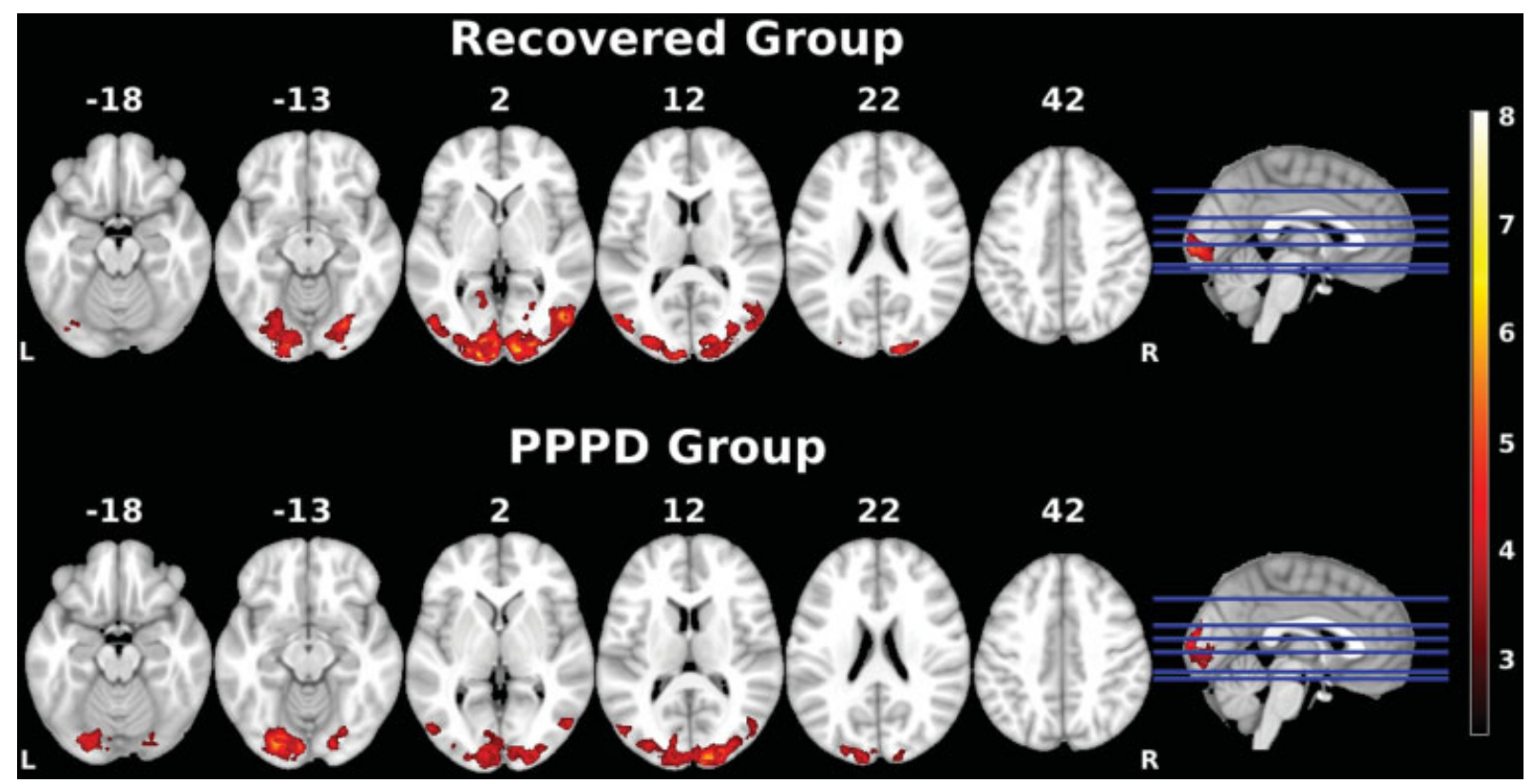

Fig. 2 Functional magnetic resonance imaging during visualization of positive blocks in recovered group (top) and persistent posturalperceptual dizziness group (bottom).

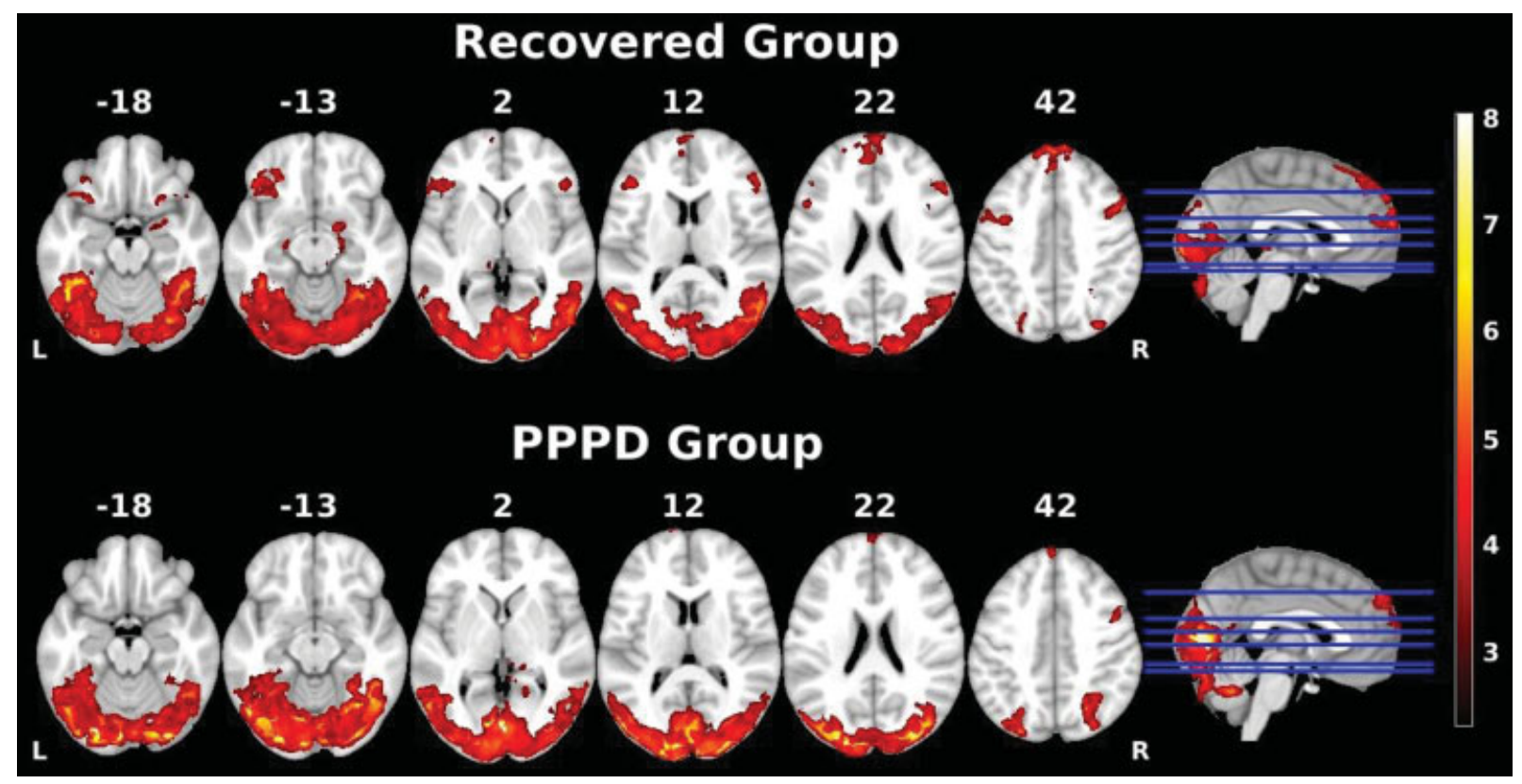

Fig. 3 Functional magnetic resonance imaging during visualization of negative blocks in recovered group (top) and persistent posturalperceptual dizziness group (bottom).

activation than the Recovered group in the left angular gyrus, which is involved in spatial orientation and cognition ${ }^{38,39}$ (-Table 3, -Fig. 4, left).

The within-group analyses of responses to negative versus positive stimuli and negative versus neutral stimuli showed that the PPPD group had little activation of the amygdala to negative or positive stimuli, whereas the Recovered group showed a pattern of amygdala activation expected from emotionallyvalenced stimuli, specifically greater to negative than to positive picture sets (-Fig. 5). ${ }^{36,37}$ The Recovered group also showed increased activity in the frontal regions closely connected to the amygdala (e.g., left and right orbitofrontal cortex), which was not seen in the PPPD group ( - Table 3). An opposite pattern was identified in two brain regions involved in space-motion processing. ${ }^{40,41}$ The PPPD group, but not the Recovered group, had increased activity in the intraparietal sulcus and parahippocampal gyrus (-Table $\mathbf{3}$ ).

\section{Discussion}

The demographics and illness characteristics of participants with PPPD indicated that our study cohort, though small in 
Table 3 Responses of brain regions to positive, negative, and neutral stimulus blocks

\begin{tabular}{|c|c|c|c|c|c|}
\hline \multicolumn{6}{|c|}{ Significant differences between groups in responses to negative versus positive stimuli } \\
\hline & $\begin{array}{l}\text { Regions } \\
\text { within clusters }\end{array}$ & $\begin{array}{l}\text { MNI } \\
\text { coordinates }\end{array}$ & $\begin{array}{l}\text { Cluster } \\
\text { size }\left(\mathrm{mm}^{3}\right)\end{array}$ & $\begin{array}{l}\text { Maximum } \\
\text { Z-value }\end{array}$ & $\begin{array}{l}\text { Cluster } \\
p \text {-value }\end{array}$ \\
\hline PPPD $<$ Recovered & Anterior cingulate cortex & $19,36,-8$ & 6,526 & 4.14 & 0.00147 \\
\hline PPPD > Recovered & Left angular gyrus & $-41,-62,46$ & 5,459 & 4.64 & 0.00503 \\
\hline \multicolumn{6}{|c|}{ Significant differences within the PPPD group in responses to negative versus positive stimuli ${ }^{* *}$} \\
\hline & Right intraparietal sulcus & $34,-59,44$ & 6,328 & 5.75 & $5.96 \times 10^{-8}$ \\
\hline \multicolumn{6}{|c|}{ Significant differences within the PPPD group in responses to negative versus neutral stimuli ${ }^{* *}$} \\
\hline & Right parahippocampal gyrus Brainstem & $18,-33,-1$ & 1,151 & 4.33 & 0.0379 \\
\hline \multicolumn{6}{|c|}{ Significant differences within the Recovered group in responses to negative versus positive stimuli ${ }^{* * *}$} \\
\hline & Left amygdala* & $-21,-3,-17$ & 2,068 & 4.74 & 0.00153 \\
\hline & Right amygdala* & $18,-1,-11$ & 1,242 & 4.85 & 0.0232 \\
\hline \multicolumn{6}{|c|}{ Significant differences within the recovered group in responses to negative versus neutral stimuli ${ }^{* * *}$} \\
\hline & $\begin{array}{l}\text { Left orbitofrontal cortex } \\
\text { Left inferiofrontal cortex } \\
\text { Left temporal pole }\end{array}$ & $-43,30,7$ & 7,272 & 5.38 & $1.20 \times 10^{-8}$ \\
\hline & Left middle frontal gyrus & $-45,8,53$ & 4,451 & 4.85 & $4.410^{-6}$ \\
\hline & $\begin{array}{l}\text { Right orbitofrontal cortex } \\
\text { Right temporal pole }\end{array}$ & $24,-22,-9$ & 4,381 & 4.68 & $5.19 \times 10^{-6}$ \\
\hline & $\begin{array}{l}\text { Right amygdala* } \\
\text { Brainstem }\end{array}$ & $39,25,-28$ & 1,535 & 4.90 & 0.0104 \\
\hline
\end{tabular}

Abbreviations: MNI, Montreal Neurological Institute; PPPD, persistent postural-perceptual dizziness.

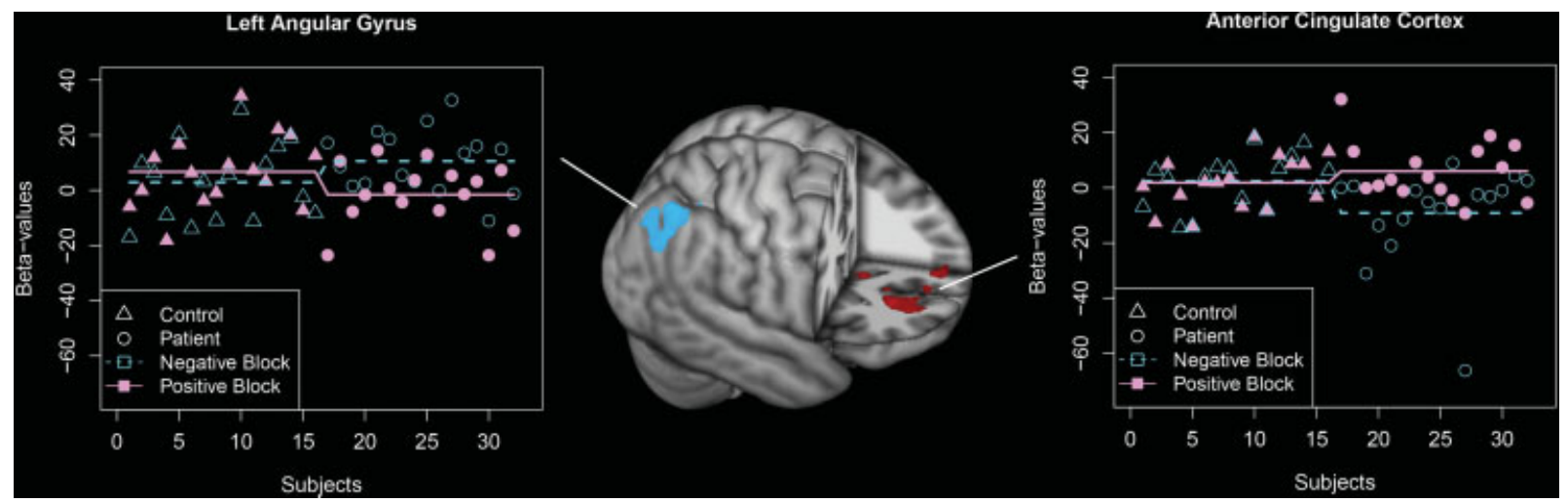

Fig. 4 Left: functional magnetic resonance imaging signal variation map during the visual paradigm in persistent postural-perceptual dizziness group versus recovered group, in the subtraction of negative from positive contrasts (negative $>$ positive). Right: functional magnetic resonance imaging signal variation map during the visual paradigm in persistent postural-perceptual dizziness group versus recovered group, in the subtraction of positive from negative contrasts (positive $>$ negative).

number, was representative of women with this condition, 2,3,33,34 and that recovered individuals offered a reasonable comparison group to control for exposure to illnesses that cause acute vertigo or dizziness.

The finding of reduced activity in the anterior cingulate cortex in the PPPD group was compatible with the fMRI results of Indovina et al, ${ }^{20}$ who found reduced responses in this region to sound-evoked vestibular stimulation in patients with CSD, and also consistent with the voxel-based morphometric study of Wurthmann et al, ${ }^{21}$ who found reduced gray matter volume of the anterior cingulate in patients with PPV, both compared with healthy controls. Thus, alterations in structure or function of the anterior cingulate cortex have been found in all three neuroimaging studies of PPPD, CSD, and PPV reported to date, identifying this as an important region for pathophysiologic processes in the brain in PPPD. Activity in the anterior cingulate also may have been affected by the mild-to-moderate levels of trait and state anxiety reported by participants with PPPD in this study, as reduced anterior cingulate responses were observed in children and adolescents with anxiety disorders and combat veterans with posttraumatic stress disorder. ${ }^{42,43}$

The absence of significant differences in activity in the amygdala in the negative versus positive and negative versus neutral contrasts in the PPPD group was contrary to expectations, particularly as the Recovered group responded in the 


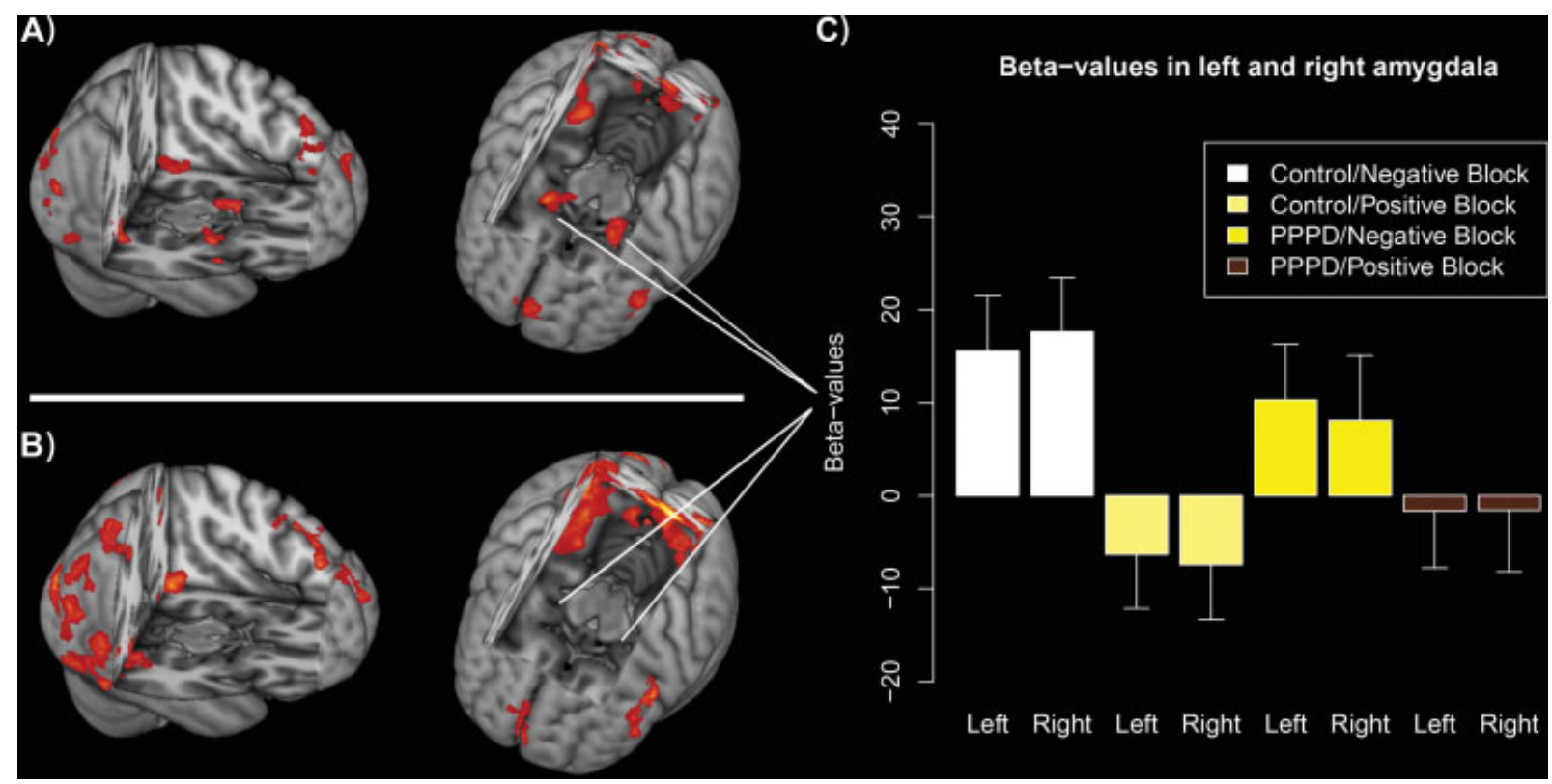

Fig. 5 (A) $\beta$-values in left and right amygdalae in recovered group (B) $\beta$-values in left and right amygdalae in persistent postural-perceptual dizziness group

expected manner, not only in the amygdala, but also in orbitofrontal and inferiofrontal cortices that regulate emotional behavior via their interconnections with the amygdala. ${ }^{44,45}$ This unexpected finding was even more striking considering that participants in the PPPD group reported mild-to-moderate anxiety and depressive symptoms. Imaging studies of patients with anxiety disorders and investigations of normal individuals using fear-conditioning paradigms have consistently found increased activity in the amygdala in response to emotionally charged stimuli with negative stimuli generating the strongest responses. $^{36,37,44}$

One explanation for these contrary results is that they were spurious, arising from problems with experimental design and procedures. However, two findings lend credence to our results. Participants in both groups were attentive and reactive to the experimental task, as demonstrated by brain activation patterns to positive and negative stimuli in occipital and frontal cortices ( - Figs. 2 and 3). Also, participants in our Recovered group demonstrated the pattern of amygdala activation expected from prior investigations of this type. $^{36,37,44}$ Thus, the unexpected results of the PPPD group were not likely due to faulty experimental factors.

A second explanation is that activity truly was suppressed in the amygdala and associated emotional processing regions in patients with PPPD. On first glance, this is difficult to reconcile with clinical research data on the multiple roles of anxietyrelated factors in PPPD $^{2,3}$ and its precursors, $4,5,7,9,11-16,28$ neuroanatomical evidence of close linkages between vestibular and anxiety systems at multiple levels in the brain, ${ }^{46}$ and neuroimaging data showing increased activity and connectivity between vestibular, visual, and anxiety regions mediated by neuroticism and introversion in normal individuals. ${ }^{18,19}$ However, it could have occurred if participants in the PPPD group responded more to spatial elements of the stimuli than their emotional content or if activity in the amygdala was suppressed by the potentially noxious nature of the visual task.

The possibility that participants with PPPD may have responded to spatial elements of the pictures is supported by our finding of activations of brain regions involved spacemotion processing within the PPPD group. The activated areas included the left angular gyrus, which is involved in translating spatial elements of external stimuli (e.g., left side-right side) into internal mental representations, ${ }^{38,39}$ the intraparietal sulcus, which contains topographic maps of visual space, ${ }^{40}$ and the parahippocampal gyrus, which supports contextual processing of spatial elements of visual scenes and navigation tasks. ${ }^{41}$ This pattern of activation of space-motion regions was not observed in the Recovered group. Therefore, even though the stimuli were stationary, our results suggest that their spatial elements were more compelling than their emotional content to participants with PPPD.

Many patients with PPPD report that their longstanding symptoms are bothersome, more than anxiety-provoking. In contrast to the fear and anxiety that patients often experience during their initial vestibular and balance symptoms, they find chronic dizziness and situations that provoke it to be frustrating and unpleasant, including tasks that require sustained visual focus on electronic screens. ${ }^{2}$ Neuroimaging research using painful stimuli found decreased activity in the amygdala, which was thought to arise from inhibitory effects of higher-level brain mechanisms during noxious tasks. ${ }^{47}$ Participants in our study were not overtly distressed by the experimental paradigm, most likely because it was short in duration ( $<10$ minutes) and did not involve particularly troublesome (e.g., moving) stimuli. However, reduced activation of the amygdala in the PPPD group could have reflected a subtle, perhaps anticipatory, reaction to the possibility that the task might be noxious. 
Taken together, these results make it difficult to conceptualize PPPD as a simple phobic condition or one in which fear conditioning has a sustaining role. Initial descriptions of both PPV and CSD invoked fear conditioning as a pathophysiologic process, ${ }^{4,8}$ but subsequent reports offered more sophisticated hypotheses. ${ }^{3}$ Brandt and Dieterich ${ }^{4,48}$ postulated that the pathophysiologic mechanism underlying PPV is a disturbance of space constancy, which involves instinctive awareness of differences between actual and desired movements and reflexive actions to correct those differences. Individuals with PPV are thought to be overly focused on even small differences, causing them to make repeated and unnecessary corrections. In the present fMRI study of PPPD and the previous one of $\mathrm{CSD}^{20}{ }^{20}$ activity in the anterior cingulate cortex was reduced. One of the roles of the anterior cingulate is to compute an error signal between predicted and observed activities to adjust attention and modify behavior as needed. ${ }^{43,49}$ Thus, reduced activity of the anterior cingulate may align with a potential problem of space constancy in PPPD.

Staab ${ }^{13}$ hypothesized that the pathophysiologic mechanism responsible for CSD is a failure of readaptation, in which patients continue to use high-threat locomotor strategies long after they are necessary. When the illnesses that trigger PPPD are active, stiffened postural control and visual dependence may be effective countermeasures for vestibular and balance symptoms, but they are not needed after the precipitants remit. The activation of visuospatial regions of the brain observed in the PPPD group in this study may have been a manifestation of persisting visual dependence, prioritizing attention to spatial elements in the visual field to identify orienting cues. When combined with the results of Indovina et $\mathrm{al}^{20}$ which showed widespread reductions in connectivity of cortical networks linking threat assessment with spacemotion information processing, the current findings support the hypothesis that higher-level mechanisms may insufficiently check reflexive actions, such as stiffened postural control and visual dependence, causing these to persist rather than reset back to normal in patients who develop PPPD. ${ }^{8,20,50}$ The hypotheses of Brandt and Dieterich ${ }^{4,48}$ and Staab ${ }^{8}$ are complementary, as space constancy may be one of the higher-level processes that is functionally impaired in patients with PPPD. ${ }^{3}$

\section{Conclusion}

The results of this fMRI investigation of the functional vestibular disorder now known as PPPD extended the findings of Indovina et $\mathrm{al}^{20}$ and were compatible with those of Wurthmann et al. ${ }^{21}$ Although some of the results were contrary to expectations, further consideration found them to support increasingly sophisticated hypotheses about the nature of PPPD, 2,3 which recognize that anxiety-related factors may confer risk for the disorder and play a role in its genesis, but emphasize unnecessarily persistent functional alterations in locomotion (i.e., stiffened posture control) and visuospatial perception (i.e., visual dependence) as likely sustaining mechanisms, possibly maintained by a breakdown in space constancy.

Limitations of this study include the small female-only cohort and the fact that some of the results were identified only within and not between groups. Future investigations will be needed to replicate these results and extend them to men and women of all ages over the entire course of PPPD, from the initial triggering events through the onset of chronic symptoms to resolution of the disorder with effective treatment. Coupling prospective imaging data with physiologic markers of stiffened postural control and visual dependence should provide a more complete picture of the underlying mechanisms of PPPD.

\section{Funding \\ Fundação de Amparo à Pesquisa do Estado de São Paulo (Grant/Award Number: '11/51266-5')}

\section{Conflict of Interests}

The authors have no conflict of interests to declare.

\section{References}

1 World Health Organization. Persistent postural-perceptual dizziness. International Classification of Diseases, ICD-11 Beta Draft (Joint Linearization for Mortality and Morbidity Statistics), available at https://icd.who.int/browse11/lm/en\#/http://id.who.int/icd/entity/2005792829. Accessed 30 September 2019.

2 Staab JP, Eckhardt-Henn A, Horii A, et al. Diagnostic criteria for persistent postural-perceptual dizziness (PPPD): Consensus document of the committee for the Classification of Vestibular Disorders of the Bárány Society. J Vestib Res 2017;27(04): 191-208. Doi: 10.3233/VES-170622

3 Dieterich M, Staab JP. Functional dizziness: from phobic postural vertigo and chronic subjective dizziness to persistent posturalperceptual dizziness. Curr Opin Neurol 2017;30(01):107-113. Doi: 10.1097/WCO.0000000000000417

4 Brandt T, Dieterich M. Phobischer Attacken-Schwankschwindel, ein neues Syndom. Munch Med Wochenschr 1986;128:247-250

5 Jacob RG, Lilienfeld SO, Furman JMR, Durrant JD, Turner SM. Panic disorder with vestibular dysfunction: Further clinical observation and description of space and motion phobic stimuli. J Anxiety Disord 1989;3:117-130

6 Bronstein AM. Visual vertigo syndrome: clinical and posturography findings. J Neurol Neurosurg Psychiatry 1995;59(05):472-476

7 Staab JP, Ruckenstein MJ. Expanding the differential diagnosis of chronic dizziness. Arch Otolaryngol Head Neck Surg 2007;133(02): 170-176

8 Staab JP. Chronic subjective dizziness. Continuum (Minneap Minn) 2012;18(5 Neuro-otology):1118-1141

9 Schniepp R, Wuehr M, Huth S, Pradhan C, Brandt T, Jahn K. Gait characteristics of patients with phobic postural vertigo: effects of fear of falling, attention, and visual input. J Neurol 2014;261(04): 738-746

10 Cousins S, Cutfield NJ, Kaski D, et al. Visual dependency and dizziness after vestibular neuritis. PLoS One 2014;9(09):e105426

11 Staab JP, Rohe DE, Eggers SD, Shepard NT. Anxious, introverted personality traits in patients with chronic subjective dizziness. J Psychosom Res 2014;76(01):80-83

12 Chiarella G, Petrolo C, Riccelli R, et al. Chronic subjective dizziness: Analysis of underlying personality factors. J Vestib Res 2016; 26(04):403-408

13 Yan Z, Cui L, Yu T, Liang H, Wang Y, Chen C. Analysis of the characteristics of persistent postural-perceptual dizziness: A clinical-based study in China. Int J Audiol 2017;56(01):33-37

14 Staab JP, Ruckenstein MJ. Which comes first? Psychogenic dizziness versus otogenic anxiety. Laryngoscope 2003;113(10):1714-1718

15 Tschan R, Best C, Beutel ME, et al. Patients' psychological well-being and resilient coping protect from secondary somatoform vertigo 
and dizziness (SVD) 1 year after vestibular disease. J Neurol 2011; 258(01):104-112

16 Godemann F, Siefert K, Hantschke-Brüggemann M, Neu P, Seidl R, Ströhle A. What accounts for vertigo one year after neuritis vestibularis - anxiety or a dysfunctional vestibular organ? J Psychiatr Res 2005;39(05):529-534

17 Heinrichs N, Edler C, Eskens S, Mielczarek MM, Moschner C. Predicting continued dizziness after an acute peripheral vestibular disorder. Psychosom Med 2007;69(07):700-707

18 Indovina I, Riccelli R, Staab JP, Lacquaniti F, Passamonti L. Personality traits modulate subcortical and cortical vestibular and anxiety responses to sound-evoked otolithic receptor stimulation. J Psychosom Res 2014;77(05):391-400

19 Riccelli R, Indovina I, Staab JP, et al. Neuroticism modulates brain visuovestibular and anxiety systems during a virtual rollercoaster task. Hum Brain Mapp 2017;38(02):715-726. Doi: 10.1002/hbm.23411

20 Indovina I, Riccelli R, Chiarella G, et al. Role of the insula and vestibular system in patients with chronic subjective dizziness: An fMRI study using sound-evoked vestibular stimulation. Front Behav Neurosci 2015;9:334

21 Wurthmann FS, Nägel S, Holle D, et al. Gray matter decrease in phobic postural vertigo-a VBM study. Klin Neurophysiol 2012; 43:119

22 Costa PT, McCrae RR. NEO Personality Inventory Revised (NEO-PI$\mathrm{R}^{\mathrm{TM}}$ ). Lutz, FL, USA: Psychological Assessments Resources, Inc.; 1992

23 Lang PJ, Bradley MM, Cuthbert BN. International Affective Picture System (IAPS): Technical Manual and Affective Ratings. http:// csea.phhp.ufl.edu/media.html

24 Zigmond AS, Snaith RP. The hospital anxiety and depression scale. Acta Psychiatr Scand 1983;67(06):361-370

25 Spielberger CD. State-Trait Anxiety Inventory: Bibliography (2nd ed.).. Palo Alto, CA: Consulting Psychologists Press; 1989

26 Beck AT, Steer RA, Brown GK. Manual for the Beck Depression Inventory-II. San Antonio, TX: Psychological Corporation; 1996

27 Jacobson GP, Newman CW. The development of the Dizziness Handicap Inventory. Arch Otolaryngol Head Neck Surg 1990;116 (04):424-427

28 Bittar RS, Lins EM. Clinical characteristics of patients with persistent postural-perceptual dizziness. Rev Bras Otorrinolaringol (Engl Ed) 2015;81(03):276-282

29 Bittar RSM, Bottino MA, Simoceli L, et al. Vestibular Impairment secondary to glucose metabolic disorders: reality or myth? Rev Bras Otorrinolaringol (Engl Ed) 2004;70:800-805

30 Bittar RSM, Santos MD, Mezzalira R. Glucose metabolism disorders and vestibular manifestations: evaluation through computerized dynamic posturography. Rev Bras Otorrinolaringol (Engl Ed) 2016;82(04):372-376

31 Thompson KJ, Goetting JC, Staab JP, Shepard NT. Retrospective review and telephone follow-up to evaluate a physical therapy protocol for treating persistent postural-perceptual dizziness: A pilot study. J Vestib Res 2015;25(02):97-103, quiz 103-104

32 Mari JJ, Williams P. A validity study of a psychiatric screening questionnaire (SRQ-20) in primary care in the city of Sao Paulo. Br J Psychiatry 1986;148:23-26
33 Centre for Functional MRI of Brain - FMRIB. Analysis Group. . Oxford, UK. http://fsl.fmrib.ox.ac.uk/fsl/fslwiki/

34 Woo CW, Krishnan A, Wager TD. Cluster-extent based thresholding in fMRI analyses: pitfalls and recommendations. Neuroimage 2014;91:412-419

35 Worsley KJ. Statistical Analysis of Activation Images. In: Matthews PM, Smith SM. Functional MRI: An Introduction to Methods. Jezzard P. 2001

36 Paulus MP. The role of neuroimaging for the diagnosis and treatment of anxiety disorders. Depress Anxiety 2008;25(04): 348-356

37 Etkin A, Wager TD. Functional neuroimaging of anxiety: a meta-analysis of emotional processing in PTSD, social anxiety disorder, and specific phobia. Am J Psychiatry 2007;164(10): 1476-1488

38 Sack AT. Parietal cortex and spatial cognition. Behav Brain Res 2009;202(02):153-161

39 Rosenthal CR, Roche-Kelly EE, Husain M, Kennard C. Responsedependent contributions of human primary motor cortex and angular gyrus to manual and perceptual sequence learning. J Neurosci 2009;29(48):15115-15125

40 Bray S, Almas R, Arnold AE, Iaria G, MacQueen G. Intraparietal sulcus activity and functional connectivity supporting spatial working memory manipulation. Cereb Cortex 2015;25(05): 1252-1264

41 Aminoff EM, Kveraga K, Bar M. The role of the parahippocampal cortex in cognition. Trends Cogn Sci 2013;17(08):379-390

42 Swartz JR, Phan KL, Angstadt M, Klumpp H, Fitzgerald KD, Monk CS. Altered activation of the rostral anterior cingulate cortex in the context of emotional face distractors in children and adolescents with anxiety disorders. Depress Anxiety 2014;31(10): 870-879

43 Shin LM, Whalen PJ, Pitman RK, et al. An fMRI study of anterior cingulate function in posttraumatic stress disorder. Biol Psychiatry 2001;50(12):932-942

44 Keightley ML, Chiew KS, Anderson JAE, Grady CL. Neural correlates of recognition memory for emotional faces and scenes. Soc Cogn Affect Neurosci 2011;6(01):24-37

45 Ochsner KN, Gross JJ. The cognitive control of emotion. Trends Cogn Sci 2005;9(05):242-249

46 Staab JP, Balaban CD, Furman JM. Threat assessment and locomotion: clinical applications of an integrated model of anxiety and postural control. Semin Neurol 2013;33(03):297-306

47 Gondo M, Moriguchi Y, Kodama N, et al. Daily physical complaints and hippocampal function: an fMRI study of pain modulation by anxiety. Neuroimage 2012;63(03):1011-1019

48 Brandt T. Phobic postural vertigo. Neurology 1996;46(06): 1515-1519

49 Paulus MP, Stein MB. An insular view of anxiety. Biol Psychiatry 2006;60(04):383-387

50 Woll J, Sprenger A, Helmchen C. Postural control during galvanic vestibular stimulation in patients with persistent perceptualpostural dizziness. J Neurol 2019;266(05):1236-1249 